\title{
Indice de primarité et différenciation génétique des populations caprines de la steppe (Arabia) et du désert (Mekatia) d'Algérie
}

\author{
E Khemici, A Lounis, M Mamou, M Sebâa-Abdelkader, A Takoucht \\ Université de Blida, Institut d'agronomie, BP 270, 09000 Blida, Algérie
}

(Reçu le 9 septembre 1994; accepté le 25 août 1995)

\begin{abstract}
Résumé - Le degré d'appartenance des 2 populations ou races caprines algériennes Arabia (steppe) et Mekatia (oasis du désert) à la catégorie population primaire (initialement population traditionnelle), taxon entre espèce sauvage et race standardisée) est mesuré par l'emploi de 2 indices récemment introduits : l'indice de primarité loci en ségrégation (IPs) et l'indice de primarité allèles au locus Agouti (IPa). Cette mesure est basée sur la considération de 11 loci à effet visible : Agouti (A), Brown (B), Frosting (Fr), Roan (Rn), Beard (Bd), Ear Length (EL), Ear Carriage (EC), Ear Curling (ECr), Horns (Ho), Hair Length (HL) et Wattles (Wa). Les résultats obtenus dans le cas des chèvres de Laghouat ( $I P s=1$ et $I P a=1)$ et dans celui de Ghardaïa $(I P s=1)$ mettent en évidence la primarité des populations étudiées. Le degré de différenciation génétique relatif aux 2 populations caprines est estimé sur la base des profils géniques visibles de 9 loci $(A, B, F r, R n, B d, E L, H o, H L, W a)$ par l'emploi de l'indice de fixation $F S T$ de Wright. Les résultats par locus traduisent un pouvoir discriminant des loci $H L$ $(F S T(i)=0,2281), B d(F S T(i)=0,1366), \operatorname{Ho}(F S T(i)=0,0124), \operatorname{Fr}(F S T(i)=0,0114)$ et $\mathrm{A}(F S T(i)=0,0111)$. En définitive, primarité et degré de différenciation génétique global peu élevé $(F S T=0,076)$ caractérisent les populations caprines Arabia et Mekatia d'Algérie.
\end{abstract}

chèvre / gènes à effet visible / Algérie / primarité

Summary - Primarity index and genetic differentiation of Algerian goat populations of Arabia (steppe) and Mekatia (desert). The degree of belonging to the primary population category (a within-species taxonomic category between wild population and standardized breed, formerly called traditional population) of the 2 Algerian goat populations or breeds Arabia (steppe) and Mekatia (oases of the desert) is calculated by the use of 2 recently introduced indices: the index of primarity loci in segregation (IPs) and the index of primarity Agouti locus (IPa). These indices consider 11 visible genetic loci: Agouti (A), Brown (B), Frosting (Fr), Roan (Rn), Beard (Bd), Ear Length (EL), Ear Carriage (EC), Ear Curling (ECr), Horns (Ho), Hair Length (HL) and Wattles (Wa). The results 
obtained show the primarity of the Arabia goat ( $\mathrm{IPs}=1$ and $\mathrm{IPa}=1$ ) and the primarity of the Mekatia goat (IPs = 1). The degree of genetic differentiation between these goat populations is estimated on the basis of the visible genetic profiles of 9 loci $(\mathrm{A}, \mathrm{B}, \mathrm{Fr}, \mathrm{Rn}$, $\mathrm{Bd}, \mathrm{EL}, \mathrm{Ho}, \mathrm{HL}, \mathrm{Wa})$ using Wright's fixation index FST. The results show the power of discrimination of the loci $\mathrm{HL}(\mathrm{FST}(\mathrm{i})=0.2281), \mathrm{Bd}(\mathrm{FST}(\mathrm{i})=0.1366)$, Ho $(\mathrm{FST}(\mathrm{i})=$ $0.0124)$, Fr $(\mathrm{FST}(\mathrm{i})=0.0114)$ and $\mathrm{A}(\mathrm{FST}(\mathrm{i})=0.011)$ It is concluded that primarity and moderate global genetic differentiation ( $\mathrm{FST}=0.076$ ) characterize the Algerian goat populations Arabia and Mekatia.

goat / visible gene effect / Algeria / primarity

\section{INTRODUCTION}

La classification évolutive des animaux de ferme proposée par Lauvergne (1982) prend en considération l'existence de 4 catégories : espèce sauvage, population traditionnelle, race standardisée et lignée sélectionnée. La catégorie population traditionnelle, renommée plus récemment population primaire (Lauvergne, 1993), est définie comme une population dérivant directement de l'espèce sauvage ancestrale par domestication, non fragmentée en isolats génétiques et sur laquelle l'homme n'a pas exercé de sélection systématique. La domestication atténue, en effet, la pression de sélection naturelle sur les mutants à effet visible, permettant leur accumulation qui génère alors une variabilité génétique visible facilement remarquable (Lauvergne, 1993). Les premières analyses quantifiées de cette variabilité dans l'espèce caprine Capra hircus L concernent des populations du bassin méditerranéen (Gruppetta et al, 1988; Renieri et al, 1988). Plus récemment, à propos de chèvres brésiliennes, 2 indices ont été introduits par Machado et al (1992); l'indice de traditionalité loci en ségrégation et indice de traditionalité allèles au locus Agouti. Ces 2 indices ont été renommés indices de primarité par Lauvergne et al (1993). D'anciens auteurs ont procédé au recensement des ressources génétiques caprines d'Afrique du Nord occultant la variabilité génétique visible manifestée par ces populations (Geoffroy Saint-Hilaire, 1919; Rivière et Lecq, 1928; Mason, 1951; Chellig, 1978). La question qui se pose alors est de savoir à quelle catégorie appartiennent les populations caprines d'Algérie. Le présent article rapporte les résultats d'une première série d'investigations utilisant les connaissances acquises en matière de génétique des caractères à effet visible chez les caprins d'une part, et d'autre part les indices de primarité précédemment cités.

Les appellations attribuées aux populations de moutons et de chèvres d'Algérie par les éleveurs traditionnels s'inspirent principalement des marqueurs génétiques visibles, comme le patron pigmentaire, de l'appartenance tribale, ou des 2 à la fois. Ainsi, la distinction des 2 populations ou races caprines étudiées exprime manifestement une différenciation génétique qui nous est apparue intéressante à quantifier par l'emploi de l'indice de fixation FST de Wright (Hartl, 1980). 


\section{MATÉRIEL ET MÉTHODES}

\section{Choix des zones d'échantillonnage}

Le cheptel caprin d'Algérie compte environ 4 millions de têtes localisées dans les zones montagneuses de l'Atlas tellien, les hautes plaines, l'Atlas saharien, la steppe et les oasis du Sahara (ministère de l'Agriculture, 1992). Les orientations émanant des travaux de Geoffroy Saint-Hilaire (1919), Rivière et Lecq (1928), Mason (1951) et Chellig (1978) sont en accord sur un point, celui de l'existence de 2 races caprines locales. La race Arabia dont l'aire d'extension s'étalerait du nord jusqu'à la limite sud de la steppe et la Mekatia qui se rencontre plus au sud, au-delà de cette limite et resterait confinée aux oasis du désert. Cette classification nous a conduits à l'échantillonnage au sein des régions de Laghouat (steppe) et Ghardaïa (oasis du désert) (fig 1), 2 régions entre lesquelles l'isohyète $100 \mathrm{~mm}$ (orienté est-ouest entre $32^{\circ}$ et $33^{\circ}$ de latitude nord) vient séparer respectivement les étages bioclimatiques sub-désertique et désertique (Seltzer, 1946).

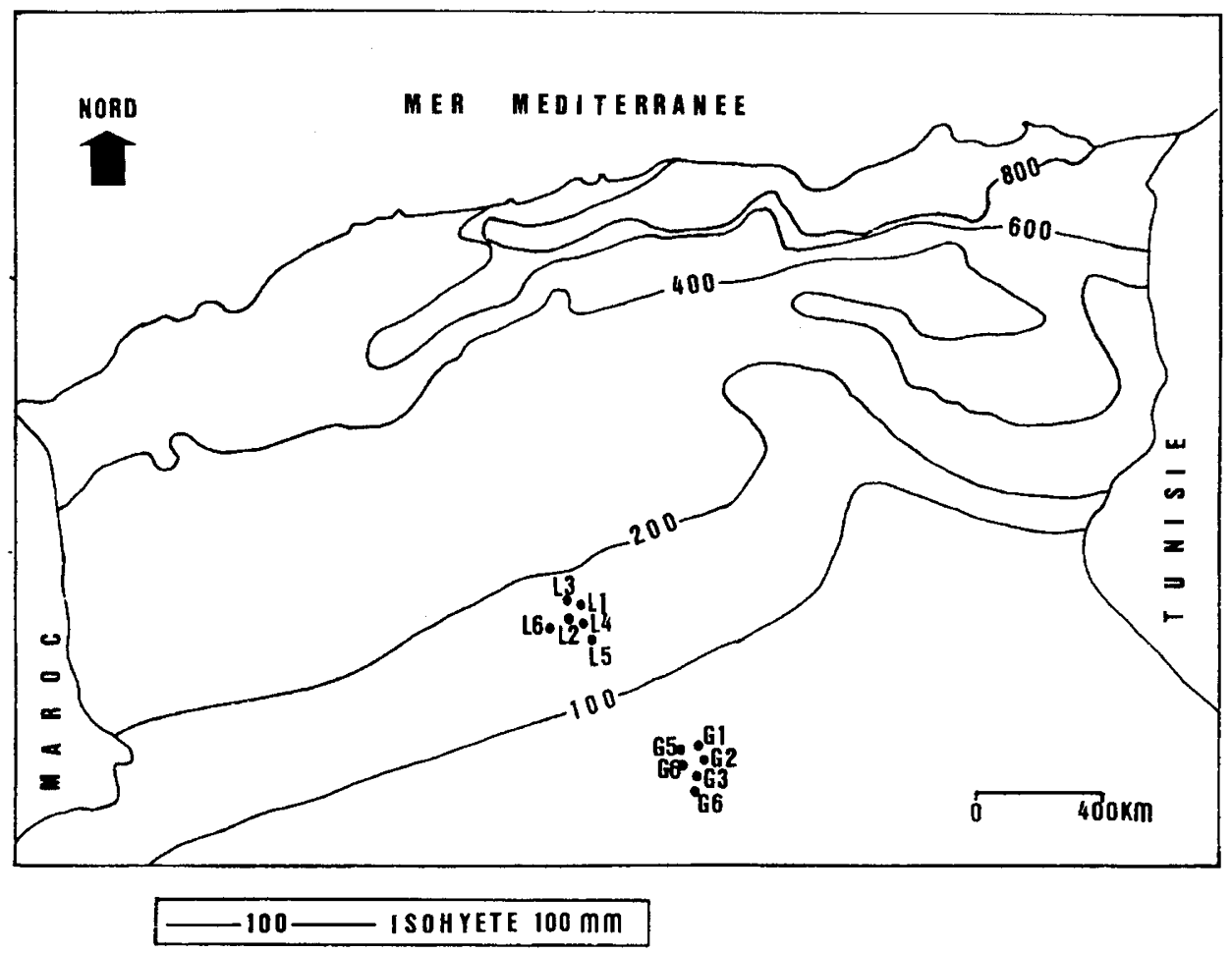

Fig 1. Localisation des sites d'échantillonnage ( $\mathrm{L}_{i}$ : échantillons de Laghouat; $\mathrm{G}_{i}$ : échantillons de Ghardaïa). 


\section{Réalisation de l'échantillonnage}

Les données recueillies au cours de sondages effectués en janvier et février 1992 proviennent d'élevages familiaux du type traditionnel et n'ont pris en considération que les animaux âgés d'au-moins une année. Les observations portent, au total, sur 353 individus ( 6 troupeaux) pour la région de Laghouat (population Arabia) (316 chèvres, 37 boucs) et sur 224 autres (6 troupeaux) pour celle de Ghardaïa (population Mekatia (205 chèvres, 19 boucs) (tableau I).

Tableau I. Effectifs des échantillons de Laghouat $\left(\mathrm{L}_{i}\right)$ et de Ghardaïa $\left(\mathrm{G}_{i}\right)$ dans les divers sites localisés sur la figure 1.

\begin{tabular}{|c|c|c|c|c|}
\hline \multirow[t]{2}{*}{ Régions } & \multirow[t]{2}{*}{ Lieux } & \multicolumn{3}{|c|}{ Effectifs } \\
\hline & & Mâles & Femelles & Total \\
\hline \multicolumn{5}{|l|}{ Laghouat } \\
\hline & Bordj-Esnoussi $\left(\mathrm{L}_{1}\right)$ & 4 & 40 & 44 \\
\hline & El-Hadjab $\left(\mathrm{L}_{2}\right)$ & 6 & 54 & 60 \\
\hline & El-Hadjab $1\left(\mathrm{~L}_{3}\right)$ & 4 & 81 & 85 \\
\hline & El-Hadjab $2-3\left(\mathrm{~L}_{4}\right)$ & 10 & 62 & 72 \\
\hline & Ijkijika $\left(L_{5}\right)$ & 11 & 65 & 76 \\
\hline & Oued M'rires $\left(\mathrm{L}_{6}\right)$ & 2 & 14 & 16 \\
\hline \multicolumn{5}{|l|}{ Ghardaïa } \\
\hline & Daïa-Bendahoua $\left(G_{1}\right)$ & 6 & 32 & 38 \\
\hline & Hadj-Messaoud $\left(\mathrm{G}_{2}\right)$ & 3 & 51 & 54 \\
\hline & Bounoura $\left(G_{3}\right)$ & 0 & 14 & 14 \\
\hline & Mermed $\left(G_{4}\right)$ & 2 & 26 & 28 \\
\hline & El-Ateuf $\left(\mathrm{G}_{5}\right)$ & 5 & 47 & 52 \\
\hline & Metlili $\left(\mathrm{G}_{6}\right)$ & 3 & 35 & 38 \\
\hline
\end{tabular}

\section{Les loci étudiés}

Onze loci à effet visible sont considérés. Quatre sont relatifs à la coloration de l'animal. Trois d'entre eux sont dialléliques : Brown $\left(B^{+}, B^{b}\right.$; wild vs brown), Frosting $\left(\mathrm{Fr}^{+}, \mathrm{Fr}^{R}\right.$; wild vs frosting), Roan $\left(\mathrm{Rn}^{+}, \mathrm{Rn}^{R}\right.$; wild vs roan) (Alexieva et al, 1990a, b; Millar et Lauvergne, 1990). Le principe de l'homologie interspécifique (Lauvergne, 1983) et les données de ségrégation relatives au quatrième locus (Agouti) responsable du patron pigmentaire (Millar et Lauvergne, 1990) permettent de tenir compte des gènes allèles suivants : nonagouti $\left(A^{a}\right)$, badgerface $\left(A^{b}\right)$, black and tan $\left(A^{t}\right)$, black and tan black belly $\left(A^{t b}\right)$, red cheek $\left(A^{r c}\right)$, mantled $\left(A^{m}\right)$, reverse mantled $\left(A^{r m}\right)$, red $\left(A^{r}\right)$ et wild $\left(A^{+}\right)$. L'allèle nonagouti est le plus récessif de la série allélique. Les autres loci concernent les caractères phénotypiques autres que ceux de la coloration : Beard $\left(B d^{+}, B d^{b}\right.$; wild vs bearded), Ear Carriage (EC $C^{+}$, $E C^{P}$; wild vs pendulous), Ear Curling $\left(E C r^{+}, E C r^{C}\right.$; wild vs curled), Ear Length ( $E L^{+}, E L^{R}$; wild vs reduced), Hair Length $\left(H L^{+}, H L^{L}\right.$; wild vs long), Horns 
$\left(\mathrm{Ho}^{+}, \mathrm{Ho}^{P}\right.$; wild vs polled), Wattles ( $\mathrm{Wa}^{+}, \mathrm{Wa}^{W}$; wild vs wattles) (COGNOSAGCOGOVICA, 1989).

\section{Mesure de la primarité}

Les indices de primarité introduits se basent sur la ségrégation observée ou non à un locus avec l'alternative $0 / 1$. Ceci élimine la surreprésentation des allèles à fréquences élevées (Lauvergne et al, 1993) et évite l'obligation d'adopter des critères permettant de considérer un locus comme étant polymorphe ou non. Ils ont, de ce fait, l'avantage de mieux apprécier l'accumulation des mutants à effet visible conséquente à la domestication.

L'indice de primarité loci à effet visible en ségrégation (IPs) a été défini comme le nombre de loci à effet visible en ségrégation $(n s)$ rapporté au nombre total de loci à effet visible $N s$ identifiés dans l'espèce (Lauvergne et al, 1993);

$$
I P s=n s / N s
$$

L'indice de primarité allèles au locus Agouti a été défini ainsi :

$$
I P a=(n a-1) /(N a-1)
$$

où $N a$ est le nombre total d'allèles en Agouti considérés et na le nombre d'allèles observés dans une population. IPa peut varier de 0 (un seul allèle en Agouti : $n a-1=0$ ) à 1 quand $n a=N a$ (Lauvergne et al, 1993).

Les troupeaux familiaux qui pâturent et transhument ensemble se caractérisent par une monte libre qui assure ainsi une situation de quasi-panmixie, critère nécessaire à l'emploi des indices de primarité. L'emploi de ces indices nécessite aussi de s'assurer qu'il ne s'agit pas d'une variabilité visible «secondaire» provenant de croisements désordonnés entre races standardisées, ce qui ne semble pas être le cas ici, vu le caractère traditionnel de l'élevage, et qu'il ne s'agit pas non plus d'une réduction de la variabilité due à des effets de dérive génique provoquant la perte du caractère de primarité (Lauvergne et al, 1993).

\section{Mesure du degré de différenciation génétique}

L'estimation du degré de différenciation génétique $(F S T)$ entre les populations caprines Arabia et Mekatia utilise les profils géniques visibles établis à partir de 9 loci $(A, B, F r, R n, B d, E L, H o, H L$ et $W a)$ sous l'hypothèse de l'équilibre de Hardy-Weinberg. L'erreur standard attribuée aux fréquences alléliques est calculée en tenant compte des formules :

$$
\left[\left(1-q^{2}\right) / 4 n\right]^{1 / 2}
$$

et

$$
[(2-p) p / 4 n]^{1 / 2}
$$

pour respectivement les allèles récessif et dominant.

Le degré de différenciation génétique $F S T(i)$ relatif à un allèle $i$ à un locus donné est obtenue selon la formule :

$$
F S T(i)=[H T(i)-\overline{H S}(i)] / H T(i)
$$


où $\overline{H S}(i)$ est la moyenne des hétérozygoties des différentes populations pour l'allèle considéré et $H T(i)$ l'hétérozygotie totale pour le même allèle sur l'ensemble $\mathrm{T}$ des populations telles que :

$$
H S(i)=2 p_{i}\left(1-p_{i}\right)
$$

et

$$
H T(i)=2 \overline{p_{i}}\left(1-\overline{p_{i}}\right)
$$

L'estimation du degré de différenciation génétique englobant l'ensemble des loci est obtenue grâce à l'emploi de la formule (Hartl, 1980) :

$$
F S T=\Sigma \sigma_{i}^{2} / \Sigma \overline{p_{i}}\left(1-\overline{p_{i}}\right.
$$

où

$$
\sigma_{i}^{2}=\overline{p_{i}^{2}}-\overline{p_{i}^{2}}
$$

Le regroupement des troupeaux de Laghouat en un seul ensemble, et de ceux de Ghardaïa en un autre, a nécessité de s'assurer de l'homogénéité statistique des distributions de fréquences alléliques dans chacun des cas à l'aide du test nonparamétrique de Kruskal-Wallis (Siegel, 1956).

\section{RÉSULTATS}

Les tableaux II et III rapportent les estimations de l'indice de primarité loci à effet visible en ségrégation (IPs) des populations caprines de Laghouat (steppe) et Ghardaïa (oasis du désert).

Dans les tableaux IV et $\mathrm{V}$ sont données les estimations de l'indice de primarité allèles au locus Agouti (IPa) de ces mêmes populations.

Les tableaux VI et VII montrent respectivement les profils géniques visibles des troupeaux de Laghouat (Arabia) et Ghardaïa (Mekatia). L'homogénéité statique des distributions de fréquences alléliques est acceptée dans le cas des échantillons de Laghouat $(p>0,05 ; \mathrm{ddl}=5)$ et dans celui de Ghardaïa $(p>0,05 ; \mathrm{ddl}=5)$.

Le tableau VIII rapporte les distributions des fréquences géniques, après regroupement des troupeaux pour chaque population, ainsi que l'estimation de l'index de fixation $F S T(i)$ (Hartl, 1980) pour chaque locus.

\section{DISCUSSION}

\section{Primarité des chèvres Arabia et Mekatia}

Les tableaux II et III montrent des IPs égaux à l'unité pour Laghouat et Ghardaïa, ce qui plaide en faveur de l'appartenance des chèvres Arabia et Mekatia à la catégorie population primaire, comme c'est le cas des chèvres du Nord Cameroun, du Tchad, de la Méditerranée orientale et occidentale, et du Brésil (Lauvergne et al, 1993; Machado et al, 1992). 
Tableau II. Indices de primarité loci à effet visible en ségrégation (IPs) identifiés dans la population caprine de Laghouat (steppe).

\begin{tabular}{lrrrrrrr}
\hline Locus & \multicolumn{7}{c}{ Lieux } \\
& \multicolumn{1}{c}{$L_{1}$} & $L_{2}$ & $L_{3}$ & $L_{4}$ & $L_{5}$ & $L_{6}$ & Total \\
\hline Agouti (A) & 1 & 1 & 1 & 1 & 1 & 1 & 1 \\
Brown (B) & 1 & 1 & 1 & 0 & 0 & 0 & 1 \\
Frosting (Fr) & 1 & 1 & 1 & 1 & 1 & 1 & 1 \\
Roan (Rn) & 1 & 1 & 1 & 1 & 1 & 1 & 1 \\
Beard (Bd) & 1 & 1 & 1 & 1 & 1 & 1 & 1 \\
Ear Length (EL) & 1 & 0 & 0 & 0 & 0 & 1 & 1 \\
Ear Carriage (EC) & 1 & 1 & 1 & 1 & 1 & 1 & 1 \\
Ear Curling (ECr) & 1 & 1 & 1 & 1 & 1 & 0 & 1 \\
Horns (Ho) & 1 & 1 & 1 & 1 & 1 & 1 & 1 \\
Hair Length (HL) & 1 & 1 & 1 & 1 & 1 & 0 & 1 \\
Wattles (Wa) & 1 & 1 & 1 & 1 & 0 & 1 & 1 \\
ns & 11 & 10 & 10 & 9 & 8 & 8 & 11 \\
Ns & 11 & 11 & 11 & 11 & 11 & 11 & 11 \\
IPs & 1,0 & 0,91 & 0,91 & 0,82 & 0,73 & 0,73 & 1,0 \\
Na & 44 & 60 & 85 & 72 & 76 & 16 & 353 \\
\hline
\end{tabular}

$n s$ : nombre de loci en ségrégation; $N s$ : nombre de loci considérés; $N a$ : nombre d'animaux par site.

Tableau III. Indices de primarité loci à effet visible en ségrégation (IPs) identifiés dans la population caprine de Ghardaïa (oasis du désert).

\begin{tabular}{lrrrrrrr}
\hline Locus & \multicolumn{7}{c}{ Lieux } \\
& \multicolumn{1}{c}{$G_{1}$} & $G_{2}$ & $G_{3}$ & $G_{4}$ & $G_{5}$ & $G_{6}$ & Total \\
\cline { 2 - 8 } Agouti (A) & 1 & 1 & 1 & 1 & 1 & 1 & 1 \\
Brown (B) & 0 & 1 & 1 & 0 & 1 & 0 & 1 \\
Frosting (Fr) & 1 & 1 & 1 & 1 & 1 & 1 & 1 \\
Roan (Rn) & 1 & 1 & 1 & 1 & 1 & 1 & 1 \\
Beard (Bd) & 1 & 1 & 1 & 1 & 1 & 1 & 1 \\
Ear Length (EL) & 0 & 0 & 0 & 0 & 1 & 0 & 1 \\
Ear Carriage (EC) & 1 & 1 & 1 & 1 & 1 & 1 & 1 \\
Ear Curling (ECr) & 1 & 1 & 1 & 1 & 1 & 0 & 1 \\
Horns (Ho) & 1 & 1 & 1 & 1 & 1 & 1 & 1 \\
Hair Length (HL) & 1 & 1 & 1 & 1 & 1 & 1 & 1 \\
Wattles (Wa) & 1 & 1 & 1 & 0 & 1 & 1 & 1 \\
ns & 9 & 10 & 10 & 8 & 11 & 8 & 11 \\
Ns & 11 & 11 & 11 & 11 & 11 & 11 & 11 \\
IPs & 0,82 & 0,91 & 0,91 & 0,73 & 1,0 & 0,73 & 1,0 \\
Na & 38 & 54 & 14 & 28 & 52 & 38 & 224 \\
\hline
\end{tabular}

$n s$ : nombre de loci en ségrégation; $N s$ : nombre de loci considérés; $N a$ : nombre d'animaux par site. 
Tableau IV. Indice de primarité allèles au locus Agouti (IPa) de la population caprine de Laghouat (steppe).

\begin{tabular}{|c|c|c|c|c|c|c|c|}
\hline \multirow[t]{2}{*}{ Allèles en Agouti } & \multicolumn{7}{|c|}{ Lieux } \\
\hline & $L_{1}$ & $\overline{L_{2}}$ & $\overline{L_{3}}$ & $L_{4}$ & $\overline{L_{5}}$ & $L_{6}$ & Total \\
\hline$A^{n a}$ & 1 & 1 & 1 & 1 & 1 & 1 & 1 \\
\hline$A^{b}$ & 1 & 1 & 1 & 1 & 1 & 0 & 1 \\
\hline$A^{t}$ & 1 & 1 & 1 & 1 & 1 & 1 & 1 \\
\hline$A^{t b}$ & 1 & 1 & 1 & 1 & 1 & 1 & 1 \\
\hline$A^{r c}$ & 0 & 0 & 0 & 1 & 0 & 0 & 1 \\
\hline$A^{m}$ & 0 & 0 & 1 & 0 & 0 & 0 & 1 \\
\hline$A^{r m}$ & 0 & 1 & 0 & 0 & 0 & 0 & 1 \\
\hline$A^{r}$ & 1 & 0 & 1 & 1 & 1 & 0 & 1 \\
\hline$A^{+}$ & 0 & 1 & 1 & 1 & 0 & 0 & 1 \\
\hline$N a$ & 9 & 9 & 9 & 9 & 9 & 9 & 9 \\
\hline$n a$ & 5 & 6 & 7 & 7 & 5 & 3 & 9 \\
\hline$I P a$ & 0,50 & 0,62 & 0,75 & 0,75 & 0,50 & 0,25 & 1,0 \\
\hline $\mathrm{Na}$ & 44 & 60 & 85 & 72 & 76 & 16 & 353 \\
\hline
\end{tabular}

$N a$ : allèles considérés; $n a$ : allèles observés; $N a$ : nombre d'animaux par site.

Tableau V. Indice de primarité allèles au locus Agouti $(I P a)$ de la population caprine de Gardaïa (oasis du désert).

\begin{tabular}{lrrrrrrr}
\hline Allèles en Agouti & \multicolumn{7}{c}{ Lieux } \\
& \multicolumn{1}{c}{$G_{1}$} & $G_{2}$ & $G_{3}$ & $G_{4}$ & $G_{5}$ & $G_{6}$ & Total \\
\hline$A^{n a}$ & 1 & 1 & 1 & 1 & 1 & 1 & 1 \\
$A^{b}$ & 1 & 1 & 1 & 1 & 1 & 1 & 1 \\
$A^{t}$ & 1 & 1 & 1 & 1 & 1 & 1 & 1 \\
$A^{t b}$ & 1 & 1 & 1 & 1 & 1 & 1 & 1 \\
$A^{r c}$ & 0 & 0 & 0 & 0 & 0 & 0 & 0 \\
$A^{m}$ & 0 & 0 & 0 & 0 & 0 & 0 & 0 \\
$A^{r m}$ & 0 & 0 & 0 & 0 & 0 & 0 & 0 \\
$A^{r}$ & 1 & 1 & 1 & 1 & 1 & 1 & 1 \\
$A^{+}$ & 0 & 1 & 1 & 0 & 1 & 1 & 1 \\
$N a$ & 9 & 9 & 9 & 9 & 9 & 9 & 9 \\
$n a$ & 5 & 6 & 6 & 5 & 6 & 6 & 6 \\
$I P a$ & 0,50 & 0,62 & 0,62 & 0,50 & 0,62 & 0,62 & 0,62 \\
$N a$ & 38 & 54 & 14 & 28 & 52 & 38 & 224 \\
\hline
\end{tabular}

$N a$ : allèles considérés; $n a$ : allèles observés; $N a$ : nombre d'animaux par site. 


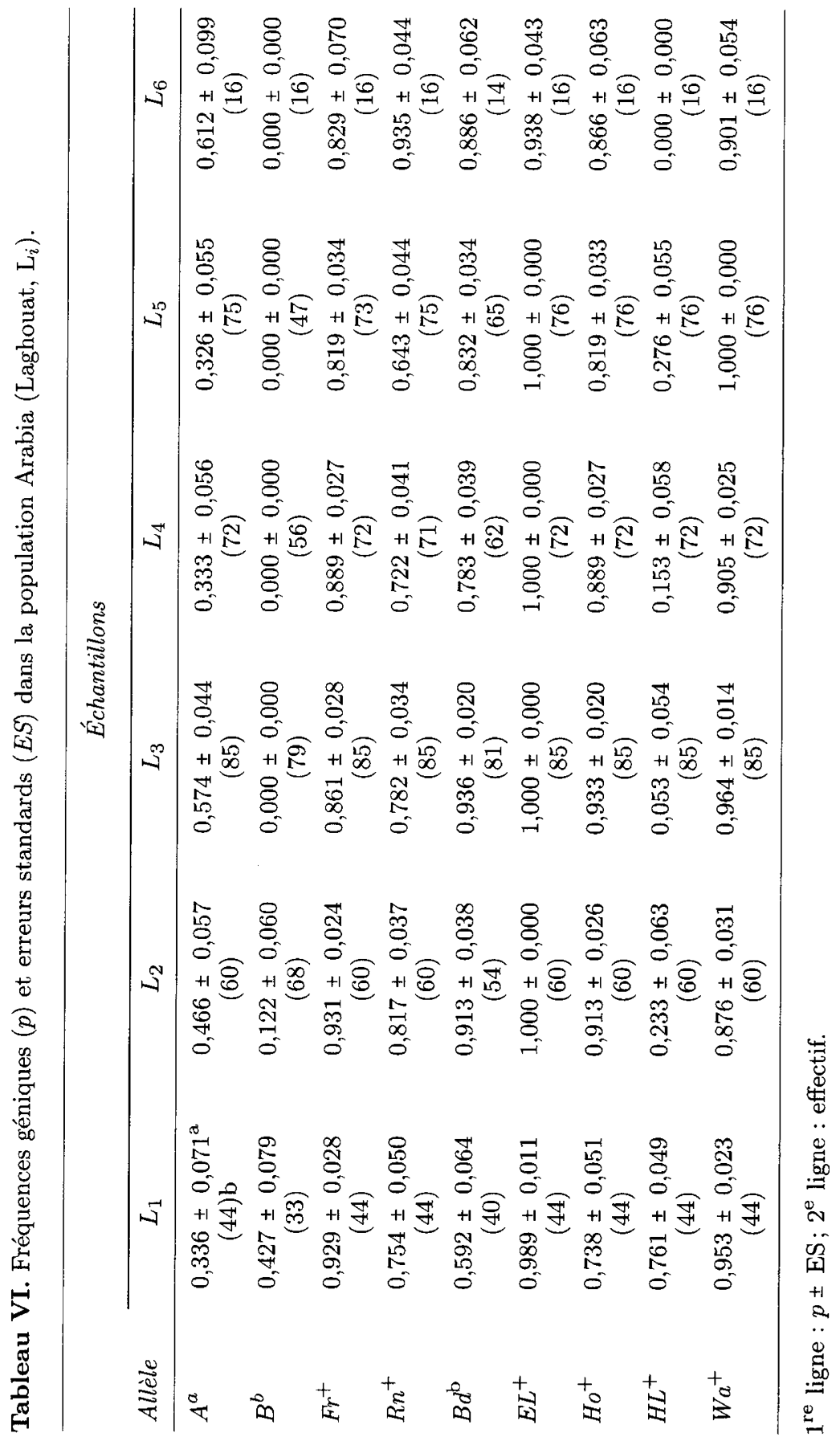




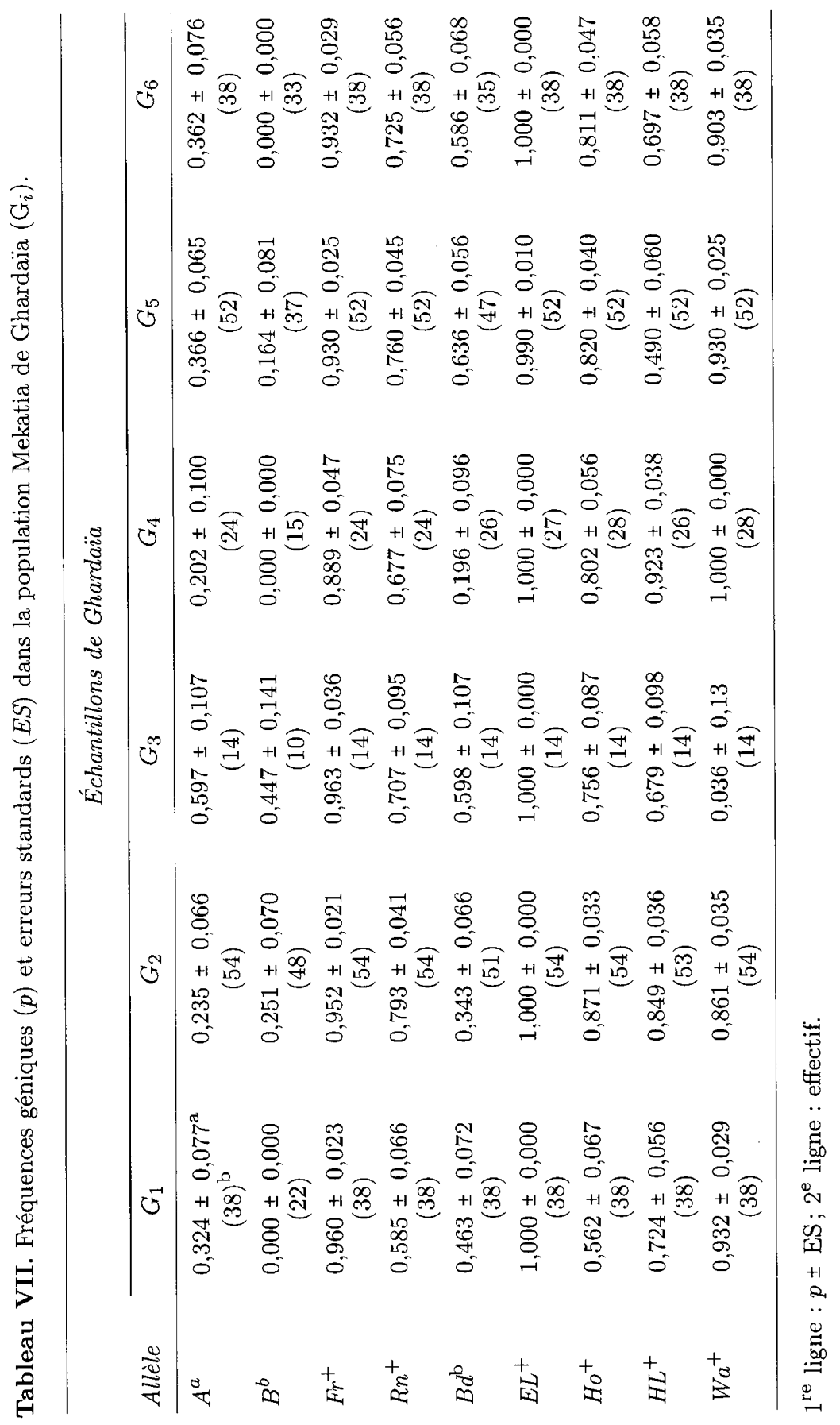


Tableau VIII. Distributions des fréquences alléliques après regroupement et indices de fixation $F S T(i)$ pour chacun des loci.

\begin{tabular}{|c|c|c|c|}
\hline & Population de Laghouat & Population de Ghardaïa & $F S T(i)$ \\
\hline \multicolumn{4}{|l|}{ Allèle } \\
\hline$A^{a}$ & $\begin{array}{l}0,504 \pm 0,027^{\mathrm{a}} \\
(260)^{\mathrm{b}}\end{array}$ & $\begin{array}{l}0,399 \pm 0,001 \\
(157)\end{array}$ & 0,0111 \\
\hline$B^{b}$ & $\begin{array}{c}0,032 \pm 0,038 \\
\quad(173)\end{array}$ & $\begin{array}{c}0,019 \pm 0,040 \\
(157)\end{array}$ & 0,0020 \\
\hline $\mathrm{Fr}^{+}$ & $\begin{array}{c}0,878 \pm 0,013 \\
(350)\end{array}$ & $\begin{array}{c}0,939 \pm 0,012 \\
(220)\end{array}$ & 0,0114 \\
\hline$R n^{+}$ & $\begin{array}{c}0,753 \pm 0,018 \\
(351)\end{array}$ & $\begin{array}{c}0,723 \pm 0,023 \\
(220)\end{array}$ & 0,0010 \\
\hline$B d^{\mathrm{b}}$ & $\begin{array}{c}0,842 \pm 0,015 \\
(316)\end{array}$ & $\begin{array}{c}0,494 \pm 0,03 \\
(205)\end{array}$ & 0,1366 \\
\hline$E L^{+}$ & $\begin{array}{c}0,996 \pm 0,002 \\
(353)\end{array}$ & $\begin{array}{c}0,998 \pm 0,002 \\
(223)\end{array}$ & 0,0000 \\
\hline $\mathrm{Ho}^{+}$ & $\begin{array}{c}0,871 \pm 0,013 \\
(353)\end{array}$ & $\begin{array}{c}0,788 \pm 0,021 \\
\quad(224)\end{array}$ & 0,0124 \\
\hline$H L^{+}$ & $\begin{array}{c}0,238 \pm 0,026 \\
(353)\end{array}$ & $\begin{array}{c}0,715 \pm 0,024 \\
(221)\end{array}$ & 0,2281 \\
\hline$W a^{+}$ & $\begin{array}{c}0,942 \pm 0,009 \\
(353)\end{array}$ & $\begin{array}{l}0,921 \pm 0,013 \\
\quad(224)\end{array}$ & 0,0016 \\
\hline
\end{tabular}

$1^{\text {re }}$ ligne $: p \pm \mathrm{ES} ; 2^{\mathrm{e}}$ ligne : effectif.

Des imprécisions relevées par Lauvergne et al (1993a) peuvent entacher l'estimation de l'IPs, comme celle relative à l'identification des loci, à l'exemple du locus de panachure blanche que nous avons évité, de ce fait, de considérer. Les fréquences basses de certains gènes tel l'allèle $E l^{R}$ au locus Ear Length ne semblent pas affecter outre-mesure le degré de primarité des chèvres Arabia et Mekatia. L'absence d'homogénéité au sein d'une population primaire susceptible de conduire à la différenciation en sous-populations peut également générer une imprécision dans la mesure de l'IPs. À ce propos, Lauvergne et al (1993b) rapportent l'existence d'un gène de réduction du cornage identifié en Afrique et son absence en Europe. Pour abonder dans le même sens, ces auteurs relèvent, aussi l'absence, en Afrique (Nord Cameroun et Tchad), du mutant $\mathrm{Ho}^{P}$ (locus Horns) qui fait disparaître les cornes et s'accompagne d'intersexualité. Il nous semble intéressant de rapporter un autre facteur susceptible de générer l'absence d'homogénéité au sein de la population primaire. Il s'agit de l'action du gène $H o^{P}$ qui s'accompagne dans les 2 sexes d'intersexualité (Ricordeau et Lauvergne, 1967; Ricordeau et al, 1972). Il semble que cette intersexualité induite par $\mathrm{Ho}^{P}$ n'existe pas au sein des populations caprines d'Algérie. En effet, tout au long de nos prospections qui concernent aussi bien les chèvres des monts Dahra et des Aurès de l'Algérie du Nord (article en préparation) que celles de la présente étude, nous avons rencontré des mâles 
et des femelles homozygotes pour $\mathrm{Ho}^{P}$ (sans cornes) reconnaissables selon Ricordeau (1972) aux protubérances osseuses arrondies du crâne, les hétérozygotes sans cornes présentant des protubérances en forme de V. Les femelles homozygotes pour le gène $H o^{P}$ qui donne l'absence de cornes devraient être atteintes de stérilité selon ce que l'on sait du comportement de ce gène dans les races européennes (Ricordeau et Lauvergne, 1967; Ricordeau, 1969; Ricordeau, 1972; Ricordeau et al, 1972). Ce que nous avons observé ne correspond pas à ce que nous devrions trouver. Nous avons, en effet, constaté l'existence de femelles allaitantes et homozygotes pour $H o^{P}$. Deux hypothèses peuvent expliquer cette situation : la distinction phénotypique des génotypes $\mathrm{Ho}^{P} / \mathrm{Ho}^{P}$ et $\mathrm{Ho}^{P} / \mathrm{Ho}^{+}$à l'origine de l'absence de cornes ne peut pas être prise en considération dans le cas des populations caprines d'Algérie ou bien $H o^{P}$ ne s'accompagne pas d'intersexualité dans ces mêmes populations. Ces hypothèses nécessitent d'être confirmées.

\section{Différenciation génétique des chèvres Arabia et Mekatia}

Les estimations de l'indice de fixation $F S T(i)$ montrent, dans leur ensemble, une apparente hétérogénéité qui exprime une différenciation sélective plus accentuée pour certains loci, alors que le FST global calculé sur l'ensemble de ces loci indique un degré de différenciation génétique faible $(F S T=0,076)$ entre les populations Arabia et Mekatia. Ainsi, le degré de différenciation génétique au locus Hair length est le plus élevé $(F S T(i)=0,2281)$. Ce fait est étroitement lié à l'appellation Mekatia. En effet, cette appellation prend sa source dans le qualificatif attribué à la pierre lisse (El-mekat) utilisée comme pavé pour les rues des oasis. L'aspect lisse que traduit l'effet des poils courts (allèle $\mathrm{Hl}^{+}$) est ainsi pris en considération pour distinguer la Mekatia des oasis, ou chèvre du M'zab, assimilée à une gazelle par les éleveurs traditionnels, de l'Arabia aux poils longs fréquente dans la steppe. Un pouvoir de discrimination caractériserait alors le comportement des fréquences alléliques au locus Hair length. Ce phénomène semble également affecter les fréquences alléliques au locus $\operatorname{Beard}(\operatorname{FST}(i)=0,1366)$ : faiblesse de la fréquence de l'allèle $B d^{b}$ (présence de barbe) $(q=0,499 \pm 0,03)$ à Ghardaïa en comparaison à la fréquence du même allèle à Laghouat $(q=0,842 \pm 0,015)$. Les estimations de l'indice de fixation $F$ indiquent, dans les cas des loci Horns $(F S T(i)=0,0124)$, $\operatorname{Fr}(F S T(i)=0,0114)$ et $\operatorname{Agouti}(F S T(i)=0,0111)$, un degré de différenciation génétique de moindre importance, mais d'égale grandeur. Nous devons, cependant, signaler la prédominance des patrons «eumélanisés » dans la steppe, exprimée par la fréquence de l'allèle $A^{a}$ (patron pigmentaire totalement noir, $q=0,504 \pm 0,027$ ), et celle des patrons "phamélanisés» dans les oasis $(q=0,399 \pm 0,001)$. La faible fréquence de l'allèle $A^{a}$ dans les oasis a d'ailleurs été relevée par Mason (1951) qui, pour qualifier la population caprine de la région en variété ou type, rapporte son existence en termes de chèvre rouge du désert. Là encore semble exister un pouvoir de discrimination des 2 populations caprines étudiées imputable à l'allèle $A^{a}$ au locus Agouti. L'homogénéité apparente des valeurs de $F S T(i)$ qui varie de 0,001 à 0,0020 pour les loci $R n, B$ et $W a$ traduit une faible hétérogénéité génétique due à ces loci entre les chèvres Arabia et Mekatia. L'estimation de l'indice de fixation $F S T(i)$ relative au locus Ear Length donne une valeur pratiquement nulle et exprime ainsi un très faible degré de différenciation génétique. En effet, la fréquence du 
gène $E l^{+}$(oreille longue) est proche de l'unité à Laghouat comme à Ghardaïa. Ce fait remarquable traduit manifestement un avantage sélectif du gène $E L^{+}$sur les aires de répartition steppique et désertique des populations caprines. Deux pressions de sélection combinées et de même sens expliqueraient cet avantage. La première, imputable à l'action des éleveurs et étroitement liée au rite du sacrifice d'Ábraham, s'exercerait en faveur du gène $E L^{+}$. Ce rite oblige en effet au sacrifice d'un animal en bonne santé dont l'oreille longue doit recouvrir l'œil qui lui est adjacent jusqu'à atteindre le chanfrein. Ainsi, les éleveurs favoriseraient le génotype $E L^{+} / E L^{+}$lors de la reproduction lui conférant un avantage sélectif. Le gène $E L^{R}$, à l'origine des oreilles rudimentaires, serait, donc par cette voie contre-sélectionné. La seconde conférerait au gène $E L^{+}$l'avantage d'un allongement de l'oreille qui constitue une défense contre la chaleur et la sécheresse en facilitant l'exportation des calories dégagées par le métabolisme par échange thermique de contact plutôt que par exportation de vapeur d'eau dans une zone sèche (Robertshaw, 1982), comme c'est le cas des populations caprines du Nord Cameroun et du Tchad (Bourzat et al, 1993). La fréquence élevée du gène $E L^{+}$témoignerait ainsi de l'adaptation aux conditions steppiques et désertiques des populations Arabia et Mekatia ou chèvre du M'zab.

\section{CONCLUSION}

Ce travail entame la cartographie des ressources génétiques caprines du Maghreb où l'absence manifeste de données en la matière est relevée comparativement aux régions nord $\mathrm{du}$ bassin méditerranéen. Il mérite d'être étendu à toute l'Algérie pour vérifier la présence d'autres populations de type primaire, vers le nord pour délimiter les zones de faible et de haute gracilité, notion récemment introduite par Lauvergne et al (1993), vers le grand sud pour préciser le pouvoir discriminant des allèles aux loci Agouti et Hair length.

\section{REMERCIEMENTS}

Nous tenons à remercier B Benbouzid, SE Nader, A Kebbal et A Touati du rectorat de l'université de Blida pour avoir autorisé et encouragé le financement de ces travaux dans le cadre du projet sur les ressources génétiques ovicaprines d'Algérie. Nous remercions également les éleveurs de Laghouat et Ghardaïa pour leurs appuis et disponibilité. Nous n'oublierons pas de remercier JJ Lauvergne du laboratoire de génétique factorielle de l'INRA de Jouy-en-Josas (France) avec lequel les contacts n'ont pas manqué d'être fructueux.

\section{RÉFÉRENCES}

Alexieva SA, Aliev GA, Bourzat D, Denis B, Dolling CHS, Lauvergne JJ, Lundie RS, Malher X, Millar P, Rachkovsky ML, Rae AL, Renieri C, Sponenberg DP, Tucker EM (1990a) Gene nomenclature in sheep and goats 1989. In : Loci for Coat Color of Sheep and Goats 1989 (JJ Lauvergne, ed) COGOVICA/COGNOSAG, Clamart, 3-7 
Alexieva SA, Aliev GA, Bourzat D, Denis B, Dolling CHS, Lauvergne JJ, Lundie RS, Malher X, Millar P, Rachkovsky ML, Rae AL, Renieri C, Sponenberg DP, Tucker EM (1990b) General procedure for listing loci and alleles of sheep and goats 1989. In : Loci for Coat Color of Sheep and Goats 1989 (JJ Lauvergne, ed), COGOVICA/COGNOSAG, Clamart, 9-13

Bourzat D, Souvenir Zafindrajaona P, Lauvergne JJ, Zeuh V (1993) Comparaison morphobiométrique de chèvres au Nord Cameroun et au Tchad. Rev Élev Med Vét Trop 4, 667-674

Chellig R (1978) La production animale de la steppe. In : Congrès sur le nomadisme en Afrique, Addis-Abbéba, 6-10 février 1978, 96-112

COGNOSAG/COGOVICA (1989) Goats allelic series 1987. Visible traits other than colour. In : Standardized Genetic Nomenclature for Sheep and Goats 1987. Loci for Visible Traits other than Color and Blood and Milk Polymorphisms (JJ Lauvergne, ed), Paris, BRG, 125-135

Geoffroy Saint-Hilaire H (1919) Espèce caprine. In : L'élevage en Afrique du Nord. MarocTunisie-Algérie (A Challamel, ed), Paris, 287-294

Gruppetta A, Renieri C, Silvestrelli M, Valfé F (1988) Profils génétiques visibles de la chèvre de Malte. In : Populations traditionnelles et premières races standardisées d'Ovicaprinæ dans le bassin méditerranéen 1986 (JJ Lauvergne, ed), Gontard/Manosque 30 juin-3 juillet 1986, colloque INRA $n^{\circ} 47$, France, 113-121

Hartl DL (1980) Genetic differentiation of populations. In : Principles of Population Genetics (Sinauer Associates, eds), Sinauer Associates Inc, Sunderland, 162-165

Lauvergne JJ (1982) Genética en poblaciones animales después de la domestication : concecuencias para la conservacion de las razas. In : Proceedings of the 2nd World Congress on Genetics applied to Livestock Production, Madrid, 4-8 October 1982, Vol 6, $77-78$

Lauvergne JJ (1983) Utilisation du principe d'homologie interspécifique pour l'étude du déterminisme de la couleur du pelage des ruminants domestiques : l'exemple du locus Agouti. Bull Soc Zool 108, 201-243

Lauvergne JJ (1993) Breed development and breed differentiation. In : Proc CEC Workshoop and Training Course (D Simon, D Buchenauer, eds), Hannover 7-9 décembre 1992, 53-64

Lauvergne JJ, Bourzat D, Souvenir Zafindrajaona P, Zeuh V, Ngo Tatia AC (1993a) Indices de primarité de chèvres au Nord Cameroun et au Tchad. Rév Élev Méd Vét Pays Trop 4, 651-665

Lauvergne JJ, Souvenir P, Minvielle F, Bourzat D, Zeuh V (1993b) Un gène de réduction de la longueur des cornes de la chèvre au Nord Cameroun et au Tchad. Rév Élev Méd Vét Pays Trop 46, 645-650

Machado T, Lauvergne JJ, Souvenir Zafindrajaona P (1992) Le scénario de peuplement caprin brésilien depuis la découverte. Arch Zootec Cordoba 41, 455-466

Mason IL (1951) Goat. In : A World Dictionary of Livestock Breeds, Types and Varieties CAB, Farnham Royal, Slough, Bucks 99-116

Millar P, Lauvergne JJ (1990) Loci for coat color of goats. In : Loci for Coat Color of Sheep and Goats 1989 (JJ Lauvergne, ed), COGOVICA/COGNOSAG, Clamart, 59-66

Ministère de l'Agriculture (1992) Comptes rendus de la consultation nationale sur l'agriculture (ministère de l'Agriculture, ed), Alger, 20-22 avril

Renieri C, Rubino R, La Tessa D, Muscillo F, Sarrica G, Zarriello G (1988) Profils génétiques visibles de la chèvre $\mathrm{du}$ sud de l'Italie. In : Populations traditionnelles et premières races standardisées d'Ovicaprinæ dans le bassin méditerranéen 1986 (JJ Lauvergne, ed), Gontard/Manosque, 30 juin-3 juillet 1986, colloque INRA $\mathrm{n}^{\circ} 47$, France, 124-134 
Ricordeau G (1969) Surprolificité des génotypes sans cornes dans les races caprines alpines Saanen, Alpine chamoisée et Poitevine. Ann Génét Sél Anim 1, 4, 391-395

Ricordeau G (1972) Distinction phénotypique des caprins homo- et hétérozygotes cornus. Ann Génét Sél Anim 4, 3, 469-475

Ricordeau G, Lauvergne JJ (1967) Hypothèse génétique unique pour expliquer la présence d'intersexués, de mâles en excès et de mâles stériles en race caprine Saanen. Ann Zootech $16,323-334$

Ricordeau G, Bouillon J, Hulot F (1972) Pénétrance de l'effet de stérilité totale lié au gène sans cornes $\mathrm{P}$ chez les boucs. Ann Génét Sél Anim 4, 537-542

Rivière C, Lecq H (1928) Bétail et élevage. In : Traité pratique d'agriculture pour le nord de l'Afrique (Société d'éditions géographiques, maritimes et coloniales, ed), Paris, Tome 1, 349-352

Robertshaw D (1982) Concepts in animal adaptation, thermorégulation of the goat. In : Proc Int Conf Goal, Goal, Production and disease, 395-397

Seltzer P (1946) Le climat de l'Algérie. Travaux de l'Institut météorologique physique globale, Alger

Siegel S (1956) The case of $\mathrm{k}$ independent samples. In : Nonparametric statistics for the behavioral sciences, McGraw-Hill, New York, 174-193 